\title{
Antigen-specific assessment of the immunological status of various groups in a leprosy endemic region
}

Angélica da Conceição Oliveira Coelho Fabri ${ }^{1,2^{*}}$, Ana Paula Mendes Carvalho ${ }^{2 \dagger}$, Sergio Araujo ${ }^{3 \dagger}$, Luiz Ricardo Goulart ${ }^{4,5 \dagger}$, Ana Márcia Menezes de Mattos ${ }^{6 \dagger}$, Henrique Couto Teixeira ${ }^{7 \dagger}$, Isabela Maria Bernardes Goulart ${ }^{3 \dagger}$, Malcolm S. Duthie ${ }^{8 \dagger}$, Rodrigo Correa-Oliveira ${ }^{2,9,10,11 \dagger}$ and Francisco Carlos Félix Lana $a^{2,12 \dagger}$

\begin{abstract}
Background: Serological tests can be important tools to assist in the diagnosis of leprosy and can contribute to an earlier diagnosis. The aim of this study was to evaluate the antibody responses against phenolic glycolipid-1 (PGL-1), natural disaccharide linked to human serum albumin via an octyl (NDO-HSA), Leprosy IDRI Diagnostic-1 (LID-1) and natural disaccharide octyl - Leprosy IDRI Diagnostic-1 (NDO-LID) in leprosy patients, household contacts of patients and the general population.
\end{abstract}

Methods: Enzyme-linked immunosorbent assays were used to analyze the antigen-specific antibody responsesof 94 leprosy cases, 104 household contacts of cases and 2.494 individuals from the general population.

Results: A positive correlation was observed for the antibody responses to all antigens studied. A higher proportion of seropositivity for all antigens, along with stronger magnitude of response, was observed in multibacillary (MB) leprosy patients and household contacts of MB leprosy patients compared with the levels observed in paucibacillary (PB) leprosy patients and household contacts of PB leprosy patients. A substantial and significant positive correlation was found between seropositivity and the bacterial index for the leprosy patients. Anti-PGL-1 tests were more frequently positive than anti-NDO-HSA tests among patients with all clinical forms of leprosy and among the group of household contacts. The LID-1 and NDO-LID antigens showed a greater capacity to identify household contacts and individuals from the general population infected with $M$. leprae.

Conclusions: Tests that measure the antibody responses against LID-1, NDO-LID, NDO-HSA and PGL-1 were effective tools for the detection of patients with MB leprosy. Our data indicate that the anti-LID-1 and anti-NDO-LID responses were more effective than an anti-NDO-HSA response for the identification of individuals with subclinical infection.

\section{Background}

Leprosy presents across a wide range of symptoms defined into 5-6 categories within the Ridley-Jopling scale, although for treatment purposes these are simplified/ consolidated to either multi- or paucibacillary (MB and $\mathrm{PB}$, respectively). The diagnosis of leprosy is not simple and, not surprisingly, many professionals have neither

\footnotetext{
* Correspondence: angelicafabri@yahoo.com.br

${ }^{\dagger}$ Equal contributors

'Department of Basic Nursing, Faculdade de Enfermagem, Universidade Federal de Juiz de Fora - UFJF, Juiz de Fora, MG, Brazil

${ }^{2}$ Postgraduate Program in Nursing, Escola de Enfermagem, Universidade

Federal de Minas Gerais - UFMG, Belo Horizonte, MG, Brazil

Full list of author information is available at the end of the article
}

the experience to recognize the various signs and symptoms of the disease nor the ability to differentiate them from other diseases [1]. Thus, leprosy patients often receive incorrect diagnoses and appropriate treatment is delayed.

Antibody responses to specific $M$. leprae antigens can be evaluated by several tests. Among these are serologic tests that measure the levels of immunoglobulin $M$ (IgM) against phenolic glycolipid-1 (PGL-1) (which may be detected by either native (anti-PGL-1) [2] or the synthetic mimetope natural disaccharide, typically linked to human serum albumin via an octyl (NDO-HSA) [3]) IgG against leprosy IDRI diagnostic 1 (LID-1) $[4,5]$ or both 
IgM and IgG against natural disaccharide octyl - leprosy IDRI diagnostic 1 (NDO-LID) [1]. The titers of antibodies against PGL-1, LID-1 and NDO-LID vary with clinical presentation, being highest in the lepromatous lepromatous (LL) clinical form and lowest, or absent, in the tuberculoid - tuberculoid (TT) form. The antibody titers generally increase as the disease progresses across the spectrum from the TT to the LL form. The bacterial index (BI) similarly varies and is positively correlated with antibody titers $[1,5,6]$.

Individuals living in leprosy endemic areas, which are typically impoverished and have high population densities, are commonly infected with M. leprae [7]. Thus, the possibility that asymptomatically infected individuals may be involved in the $M$. leprae transmission chain should not be overlooked [8]. Subclinical M. leprae infection in endemic populations is traditionally assessed using either enzyme-linked immunosorbent assays (ELISA) or lateral flow point-of-care (POC) tests to detect specific antibodies $[8,9]$. When used in this way these assays may enable earlier identification and treatment of patients, and thus contribute to both the prevention of physical disabilities and the reduced transmission of M. leprae [4].

It has been demonstrated in various settings that household contacts of untreated MB patients experience greater exposure to $M$. leprae than the general population (GPop) [6]. Anti-PGL-1 seropositivity in household contacts of leprosy patients has been associated with an increased risk of developing disease [9]. It is important, however, to consider that individuals living in highly endemic regions may be routinely exposed to $M$. leprae even if they do not live with a recognized patient. It therefore becomes pertinent to understand the performance of tests involving the PGL-1, LID-1, NDO-LID and NDO-HSA antigens in different contexts and groups within the population. The aim of this study was to evaluate serum antibody responses against PGL-1, NDO-HSA, LID-1 and NDO-LID in patients diagnosed with leprosy, in household contacts of leprosy patients and among the general population of a leprosy endemic region municipalities with varying leprosy detection rates.

\section{Methods}

\section{Patient and contact samples}

Untreated patients $(n=94)$ and household contacts $(n=104)$ were recruited at the National Reference Centre for Sanitary Dermatology and Leprosy (CREDESH), Uberlandia, Minas Gerais, Brazil, a public health care facility in an endemic region where routine prevention, including $\mathrm{Ba}-$ cillus Calmette-Guérin (BCG) vaccination, household contact monitoring, early case detection, and treatment are available and under constant supervision. The Uberlândia municipality had detection rate of 10.81/ 100.000 inhabitants in 2012 [10].
Leprosy patients were diagnosed after thorough dermato-neurological and laboratory examinations, and classified according Ridley-Jopling five-group system of clinical manifestations into: tuberculoid (TT), borderline tuberculoid (BT), mid-borderline (BB), borderline lepromatous (BL) or lepromatous (LL) [11]. For treatment purposes patients were also stratified into paucibacillary (PB), with up to five skin lesions and a negative bacilloscopy, or $\mathrm{MB}$, with more than five lesions and/or positive bacilloscopy in accordance with the World Health Organization operational classification [12].

Household contacts (HHC) who resided with leprosy patients, or had resided with leprosy patients in the five years prior to diagnosis, were examined for signs or symptoms that were suggestive of leprosy by physicians with specialized leprosy training. Most HHC were relatives of their index case (spouse, parent or sibling). HHC were stratified according to the operational and clinical classifications of their index case.

\section{Samples from the general population}

Individuals from the general population (GPop; $\mathrm{n}=$ 2.494) were selected randomly from seven municipalities in the microregion of Almenara, Minas Gerais, Brazil [13], which had a mean detection rate of 31.32/ 100.000 inhabitants in 2012 [10]. Finger-prick blood spots were collected on Whatman 3 MM paper (Whatman, Maidstone, UK) and stored at $4{ }^{\circ} \mathrm{C}$ until serum was eluted by adding $1 \%$ bovine serum albumin in $1 \mathrm{X}$ phosphate buffered saline. Based on estimates of the volume of whole blood in a $2.5 \mathrm{~mm}$ filter paper disc, the final dilution of the eluted serum was 1:100 [14, 15].

\section{Antibody detection}

Antigen-specific IgM and IgG antibodies were measured by indirect enzyme-linked immunosorbent assays (ELISA) as previously described [3]. Briefly, the four antigens (PGL-1, NDO-HSA, LID-1 and NDO-LID) were used to coat 96-well microtiter plates (LABWARE Manufacture CO, People's Republic of China); $1 \mu \mathrm{g} / \mathrm{mL}$ of LID-1 or $0.2 \mu \mathrm{g} / \mathrm{mL}$ of NDO-HSA, NDO-LID or PGL- 1 was added per well in $100 \mu \mathrm{L}$ of $0.1 \mathrm{M}$ sodium carbonate/bicarbonate buffer, $\mathrm{pH}$ 9.6, and incubated at $4{ }^{\circ} \mathrm{C}$ overnight. The assay was performed using serum samples at a dilution of 1:300 and whole blood samples at a dilution of 1:100 [14, 15]. After blocking for $1 \mathrm{~h}$ at $37{ }^{\circ} \mathrm{C}$, detection antibodies were similarly incubated for $1 \mathrm{~h}$ at $37^{\circ} \mathrm{C}$, after which four washes were performed. The wells were then treated with ortho-phenylenediamine (OPD) substrate, and the absorbance at $492 \mathrm{~nm}$ was obtained using a spectrophotometer plate reader (Molecular Devices, Sunnyvale, CA). To minimize inter- and intra-test errors, an ELISA index (EI) was calculated as follows: the EI equals the optical density (OD) of the sample divided by the OD of the cut-off [5]. 
The cut-off was calculated as an average of three controls negative (individuals living in low endemic areas leprosy and which showed result of the ML Flow test negative) plus three times the standard deviation, such that samples with EI values of 1.1 or above were considered to be positive.

\section{Statistical analysis}

Graphs and mean values were generated using GraphPad Prism version 5 (GraphPad Software Inc., La Jolla, CA, USA), and statistical analysis was performed using Statistical Package for the Social Sciences (SPSS) version 18 (SPSS Inc., Chicago, IL, USA). Statistical significance was assessed using nonparametric methods, with the Kruskal-Wallis one-way $(\mathrm{H})$ analysis of variance used to make comparisons among multiple groups and the Mann-Whitney $U$ test with Bonferroni correction used to make comparisons between two groups. Spearman's coefficient (rho) was used to test the strengths of correlations. Results were considered statistically significant when $p$-values $\leq 0.05$ were obtained or when $p$-values $\leq 0.0167$ using the Mann-Whitney $U$ test. The concordance among the four antigens was calculated using the Kappa coefficient. The NDO-HSA antigen was selected as the reference antigen because it was tested in three groups (patients, HHC and GPop) and because it is one of the most common antigens used in tests for $M$. leprae. Kappa values and their interpretation varied as follows: $<0$, no agreement; $0-0.20$, poor agreement; 0.21 0.40 , fair agreement; 0.41-0.60, moderate agreement; 0.61-0.80, substantial agreement; and 0.81-1.00, almost perfect agreement [16].

\section{Ethics statement}

This study conforms to the Declaration of Helsinki and was reviewed and approved by Research Ethic Committee (CEP) of the Federal University of Uberlandia, Protocol Number CEP/UFU 138/08, and the Research Ethic Committee (COEP) of the Federal University of Minas Gerais, Protocol Number ETIC 158/09. All participants signed an informed consent form and authorized the collection of the samples. The informed consent form for children under 18 years of age was signed by either a parent or a legal guardian.

\section{Results}

\section{Antibody responses among leprosy patients}

We determined the presence of antigen-specific antibodies in the serum samples from various groups. In the group of patients, as anticipated the seropositivity of antibodies against LID-1, NDO-LID, NDO-HSA and PGL-1 were highest in $\mathrm{MB}$ patients and lowest in $\mathrm{PB}$ patients (BT and TT forms). Antibodies against NDO-LID (28.3 \%) and PGL-1 (33.3 \%) were, however, better able to detect PB patients than both LID-1 (10.5\%) and
NDO-HSA (23.3 \%) (Table 1). Further analyses within the context of the Ridley-Jopling scale demonstrated that $100 \%(\mathrm{n}=14)$ LL patients were seropositive for antiNDO-LID and anti-PGL-1, while $85.7 \%(\mathrm{n}=12)$ and $92.9 \%(\mathrm{n}=13)$ were seropositive for anti-LID-1 and anti-NDO-HSA, respectively. Notably, the seropositivity rates for antibodies against the 4 antigens evauated were significantly different between the TT and LL groups (Fig. 1). The NDO-LID antigen had a higher seropositivity rate than LID-1 and NDO-HSA in the BT group. In the BL group the LID-1 antigen had a higher seropositivity rate than NDO-LID, and in LL group PGL-1 was observed to have a higher seropositivity than NDO-LID (Fig. 2). In addition, a positive correlation was observed for the bacterial indices (BI) and the antibody titers against LID-1 (rho $=0.81)$, NDO-LID (rho $=0.67)$, NDOHSA (rho $=0.60)$ and PGL-1 (rho $=0.61)($ all $p<0.0001)$. Thus, our data indicate that EI increase with BI and across the disease spectrum.

\section{Responses of household contacts (HHC) and the general population (GPop)}

It is documented that $\mathrm{HHC}$ are at higher risk of $M$. leprae infection and development of disease than the general population. We therefore analyzed the antigenspecific antibody responses of $\mathrm{HHC}$ and a random selection of residents (GPop) within the leprosy endemic region of Minais Gerais. The seropositivity rate was higher among the $\mathrm{HHC}$ of $\mathrm{MB}$ patients than those of $\mathrm{PB}$ patients, supporting the hypothesis that $\mathrm{HHC}$ of $\mathrm{MB}$ patients are exposed and more likely to be infected with $M$. leprae (Table 1). Positive responses were also detected among the GPop. Together, these data suggest a large proportion may be harboring $M$. leprae without any clinical symptoms of disease.

\section{Relationship of responses}

Significant differences were observed between the EI for antibodies to NDO-LID and NDO-HSA in the patient, HHC and GPop. It is noteworthy that for NDO-LID and

Table 1 Proportion of seropositivity for antibodies against LID-1, NDO-LID, NDO-HSA and PGL-1 according to groups of individuals

\begin{tabular}{|c|c|c|c|c|c|c|}
\hline & & \multirow[b]{2}{*}{ No. of samples } & \multicolumn{4}{|c|}{ No. (\%) positive ${ }^{a}$} \\
\hline & & & LID-1 & NDO-LID & NDO-HSA & PGL-1 \\
\hline \multirow[t]{2}{*}{ Patients } & $M B$ & 43 & $34(89.5)$ & $38(71.7)$ & $33(76.7)$ & $38(66.7)$ \\
\hline & PB & 51 & $4(10.5)$ & $15(28.3)$ & $10(23.3)$ & 19(33.3) \\
\hline \multirow[t]{2}{*}{$\mathrm{HHC}$} & $M B^{b}$ & 81 & $35(43.2)$ & $31(38.3)$ & $10(12.3)$ & $14(17.3)$ \\
\hline & $\mathrm{PB}^{\mathrm{C}}$ & 23 & $6(26.1)$ & $6(26.1)$ & $0(0.0)$ & $1(4.3)$ \\
\hline GPop & & 2494 & 74(21.9) & $995(41.2)$ & $191(7.9)$ & - \\
\hline
\end{tabular}

${ }^{a}$ Test were considered positive when a distinct band was observed (scored as El of 1.1 or greater)

${ }^{\mathrm{b}} \mathrm{HHC}$ of patients classified as MB

${ }^{\mathrm{C}} \mathrm{HHC}$ of patients classified as PB 


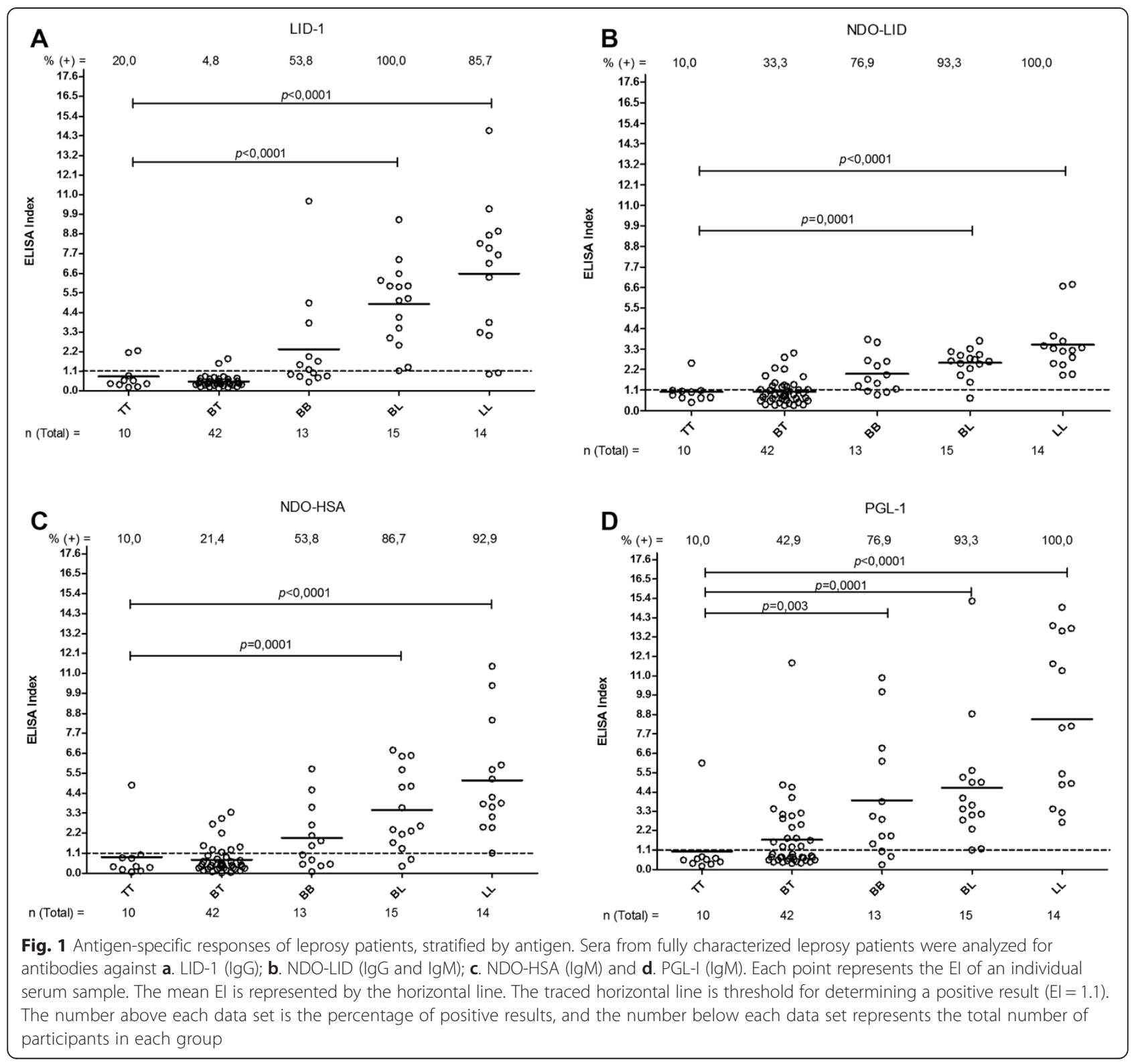

NDO-HSA the mean EI of GPop was higher than that of HHC. A significant difference was also observed between the patient group and HHC for PGL-1 (Fig. 3).

The concordance between the anti-NDO-HSA and antiLID-1 $(\mathrm{k}=0.63)$, anti-PGL-1 $(\mathrm{k}=0.62)$ and anti-NDO-LID $(\mathrm{k}=0.79)$ was substantial and significant in the patient group (all $p<0.0001$ ). In GPop the agreement was poor, while in HHC fair agreement was observed between the result of antibodies against NDO-HSA and NDO-LID $(\mathrm{k}=0.32 ; p<0.0001)$.

Using the Spearman test, a significant positive correlation was identified between the different antigens tested in GPop as well as in the patients. In HHC, no significant correlation was identified for any combination with the PGL-1 antigen (Table 2). We observed that the correlation was higher in the group of patients.

\section{Discussion}

We examined the presence of antibodies against particular M. leprae antigens among various groups living in a leprosy endemic region. Consistent with previous observations $[1,5,6]$, positive correlations with patient $\mathrm{BI}$ were observed for all of the antigens. Moreover, higher seropositivity was observed for anti-LID-1, anti-NDOLID, anti-NDO-HSA and anti-PGL-1 in MB patients (BL, LL and some BB patients) and in $\mathrm{HHC}$ of MB patients than in $\mathrm{PB}$ patients and their HHC. A large proportion of the general population were also found to be 


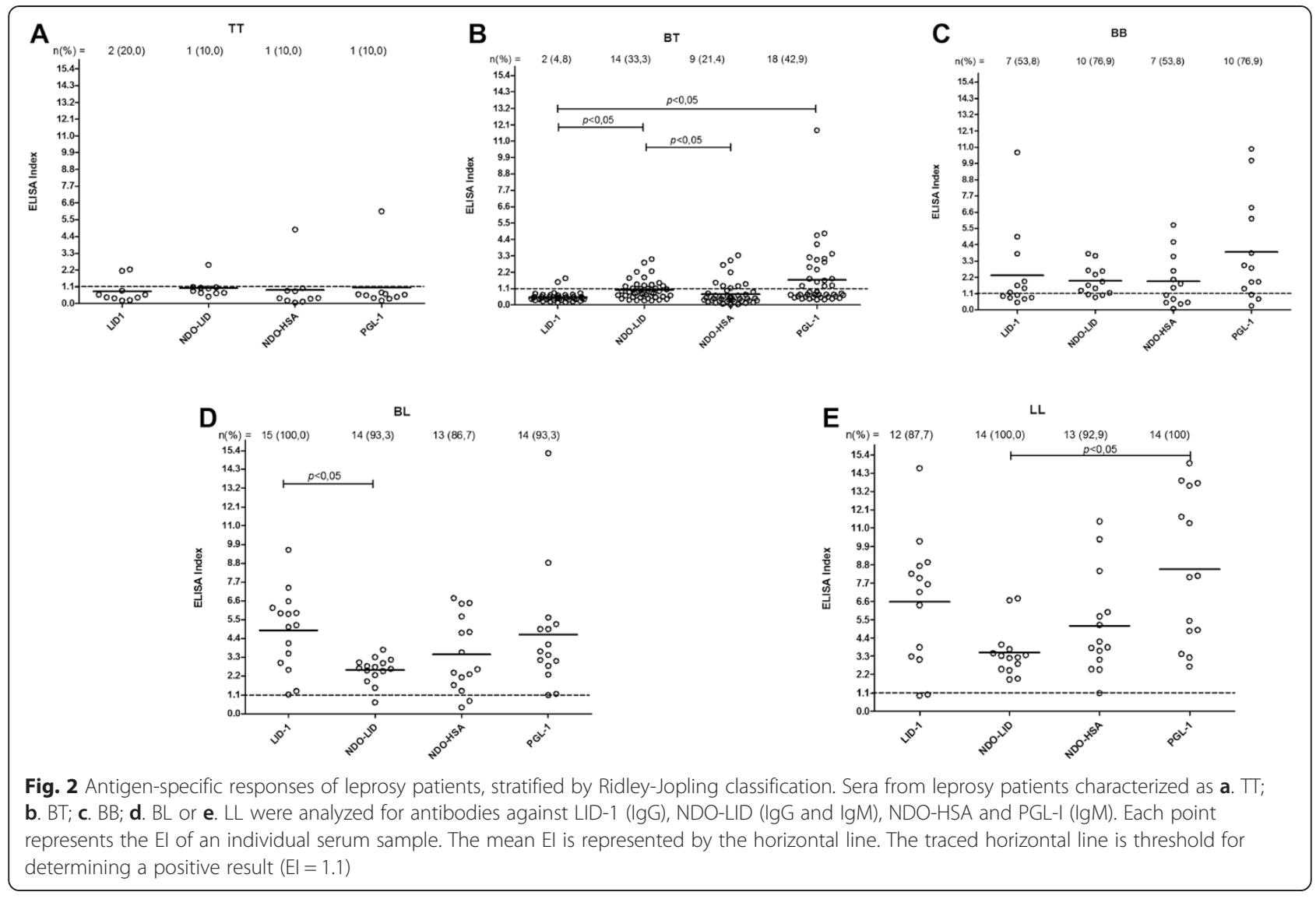

seropositive. Together, our data suggest that a relatively large proportion of the population may harbor M. leprae infection.

In agreement our findings, an earlier comparison of antibodies against either native PGL-1 or semisynthetic NDO-HSA demonstrated significant agreement between these assays [3]. Our evaluations expand this observation and identify significant agreement across patient groups for seropositivity against the LID-1, NDO-LID, NDOHSA and PGL-1. In contrast, such agreements were not observed in $\mathrm{HHC}$ or within the general population. We speculate that agreement in the antigen-specific antibody responses is observed in $\mathrm{MB}$ patients because they present with a high bacterial load and a more developed and defined humoral response. Of note, antibody responses to $M$. leprae appear to develop heterogeneously even in the controlled setting of experimental infection of armadillos [17], and thus, antigen-specific responses may develop at differing rates and be variable in infected individuals who have not yet exhibited signs of disease.

Some of the assays described here, for example ELISA detecting antibodies against the NDO-LID conjugate, appear to be more sensitive than others in the general population. The positive correlation observed for antibodies to the LID-1, NDO-HSA and NDO-LID antigens in patients, $\mathrm{HHC}$ and the general population infer that although the tests do not show perfect agreement, the value of the EI of a particular individual has a unidirectional trend.

The NDO-LID conjugate has previously demonstrated a greater capacity to identify patients with $\mathrm{MB}$ leprosy than the either of the NDO-HSA and LID-1 antigens alone [1]. Although serological tests appear to have a limited ability to aid the diagnosis of $\mathrm{PB}$ patients, our data also identified that a greater number of $\mathrm{PB}$ patients were seropositive for antibodies against NDO-LID than against either NDO-HSA or LID-1 alone, as has been reported previously $[1,6,18]$. This was especially true for patients categorized into the BT group, an effect similarly observed in a study of rapid diagnostic tests (NDO-LID, Orange Life ${ }^{\circledast}$ ) [6]. These findings suggest the possibility of using NDO-LID-based tests within leprosy control programs to identify patients early in the clinical spectrum of leprosy and to identify $\mathrm{PB}$ patients.

Our data indicate that tests detecting antibodies to PGL-I and/or LID-1 represent effective tools for the detection of $\mathrm{MB}$ patients. Among all clinical forms of leprosy and among $\mathrm{HHC}$ the use of native PGL-1 resulted in a higher positivity rate than the synthetic mimetic of 


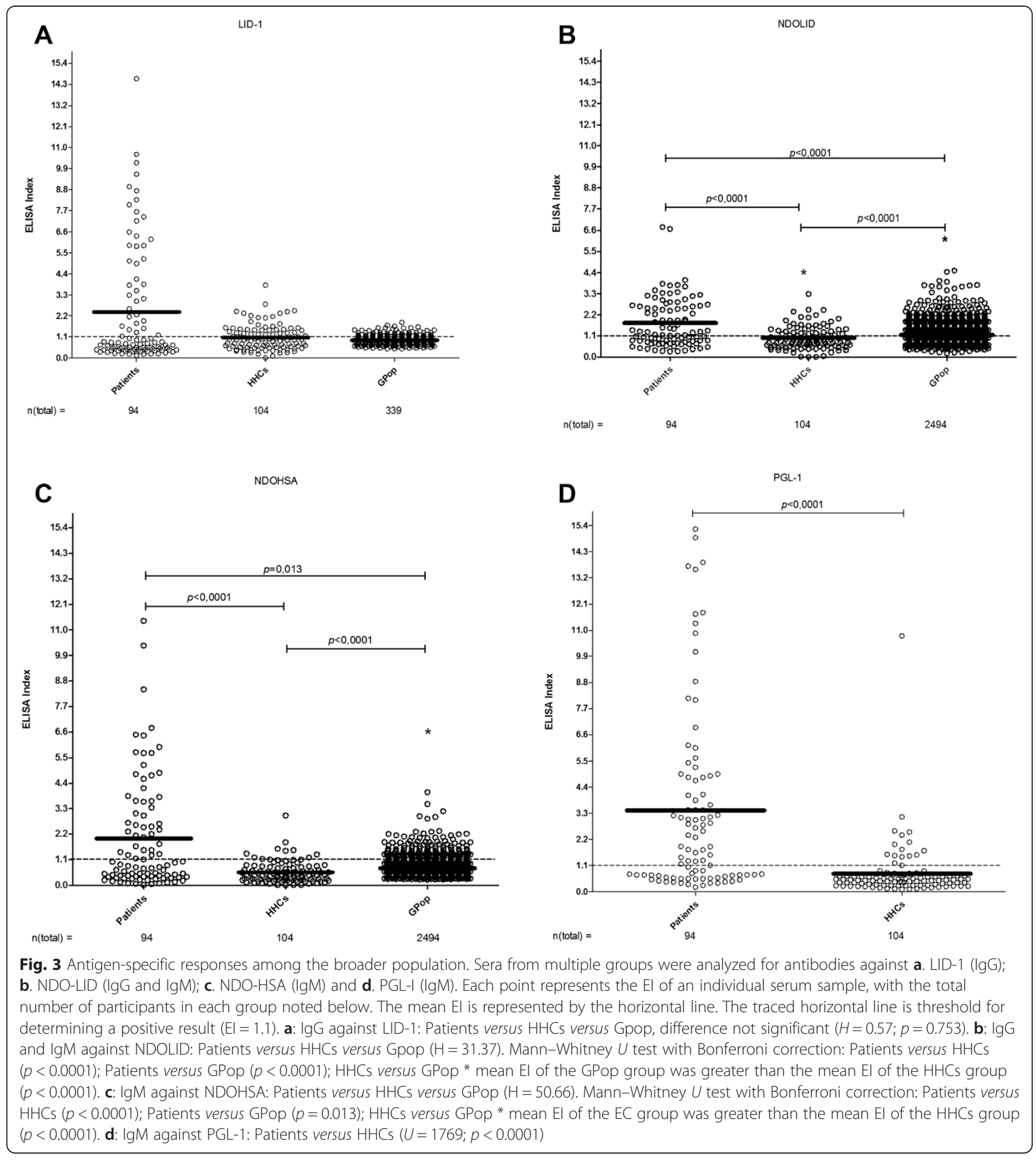

PGL-1 (NDO, conjugated to the inert carrier protein HSA). This result is in agreement with the literature, as a study comparing ELISA involving PGL-1 or NDOHSA with the ML Flow rapid test indicated that native PGL-1 ELISA resulted in greater sensitivity and accuracy than either the NDO-HSA ELISA or ML Flow tests [3]. In our comparison of leprosy patients a higher mean EI was observed for PGL-1 than NDO-LID and LID-1.
However, the need to extract native PGL-1 from the organs of $M$. leprae-infected armadillos is limiting and the sustainable use of synthetic NDO is preferred [19]. Relative to the NDO-HSA antigen, the NDO-LID conjugate demonstrated a greater potential to identify infected $\mathrm{HHC}$ and individuals from the general population who were infected with $M$. leprae. These tools could also be used to aid clinicians lacking expertize in leprosy 
Table 2 Results of the Spearman test in different groups of individuals according to antigen

\begin{tabular}{|c|c|c|c|c|c|c|}
\hline \multirow[b]{2}{*}{ Antigens } & \multicolumn{2}{|l|}{ GPop } & \multicolumn{2}{|l|}{$\mathrm{HHC}$} & \multicolumn{2}{|c|}{ Patients } \\
\hline & rho & p-value & rho & p-value & rho & $\mathrm{p}$-value \\
\hline LID-1 and NDO-LID & 0.635 & $<0.0001$ & 0.345 & 0.0003 & 0.816 & $<0.0001$ \\
\hline LID-1 and NDO-HSA & 0.506 & $<0.0001$ & 0.434 & $<0.0001$ & 0.726 & $<0.0001$ \\
\hline NDO-LID and NDO-HSA & 0.323 & $<0.0001$ & 0.864 & $<0.0001$ & 0.875 & $<0.0001$ \\
\hline PGL-1 and NDO-LID & - & - & 0.150 & 0.129 & 0.727 & $<0.0001$ \\
\hline PGL-1 and NDO-HSA & - & - & 0.134 & 0.097 & 0.718 & $<0.0001$ \\
\hline LID-1 and PGL-1 & - & - & -0.122 & 0.219 & 0.646 & $<0.0001$ \\
\hline
\end{tabular}

rho: Spearman coefficient

LID-1: Leprosy IDRI diagnostic 1

NDO-LID: Natural disaccharide octyl - leprosy IDRI diagnostic 1

NDO-HSA: Natural disaccharide linked to human serum albumin via an octyl

PGL-1: Phenolic glycolipid-1

diagnosis in general health care services to identify individuals at high risk of developing leprosy.

There is an epidemiological need for applicable diagnostic tools to detect asymptomatic individuals infected with $M$. leprae and those patients with early manifestations of leprosy. The periodic evaluation of seroprevalence rates in leprosy endemic areas may also be a useful measure of $M$. leprae transmission [20]. We expected to observe a greater positivity among HHC than in the general population because $\mathrm{HHC}$ are thought to be regularly exposed to M. leprae up until the patient is undergoing treatment [21]. On the contrary, however, we observed a higher rate of anti-NDO-LID and anti-NDO-HSA positivity in the general population than $\mathrm{HHC}$, although a limitation of the study design was that the general population was from a different region than the patients and HHC. The higher endemicity rate in the region from which the general population samples were collected probably contributed to the higher seroposivity rate and leads us to suggest that that population is regularly being exposed to $M$. leprae and suggests that the hidden prevalence of leprosy may be quite high [22].

Serological tests involving synthetic PGL-1 antigen are used in some regions to identify individuals, especially HHC, infected with $M$. leprae $[8,9]$. Our findings support the use of tests evaluating the anti-NDO-LID response, especially among $\mathrm{HHC}$ and in the general population of areas of high endemicity for leprosy. The application of anti-NDO-LID tests in the general population could assist leprosy control programs by allowing simple identification of a larger number of individuals infected with $M$. leprae and consequently those that have high risk of developing leprosy. Of particular relevance for the practicalities of large scale surveillance programs, our analysis of the general population examined whole blood collected on filter paper. A previous study demonstrated that samples stored in filter paper allowed for the recovery of antibody levels similar to those in serum samples [14] and that there is a close correlation between studies using venipuncture samples captured on filter paper [23, 24]. It is believed, therefore, that analysis of samples collected on filter paper was not a detrimental or limiting factor for our study.

The high positivity in the general population for antibodies to all of the studied antigens should raise concern because multiple reports suggest that individuals with subclinical $M$. leprae infection may be a potential transmission source for the infection of additional people [8]. As suggested by others, we support the georeferencing of patients and M. leprae-infected individuals to identify population clusters that might benefit from greater leprosy-specific vigilance by general health services. Furthermore, the quantitative measurement of antibodies against these antigens would appear beneficial in the selection of seropositive individuals for clinical examination and the earlier detection of leprosy cases.

\section{Conclusions}

We observed high rates of seropositivity for antibodies against LID-1, NDO-LID, NDO-HSA and PGL-1 antigens among various groups within the population. High rates were observed for all antigens in $\mathrm{MB}$ patients and $\mathrm{HHC}$ of MB patients, and a positive correlation was observed between serology and BI; marked, significant agreement was found between these measures in leprosy patients, in particular, but a positive correlation was also observed in the general population.

The LID-1 and NDO-LID antigens showed a greater capacity to identify $\mathrm{HHC}$ and individuals of the general population infected with $M$. leprae. PGL-1 resulted in a higher degree of positivity than NDO-HSA for all clinical forms of leprosy and for HHC.

We suggest that NDO-LID represents an important antigen for the surveillance of HHC and of the general population. Our data provide evidence that all of the antigens tested are relevant tools to support the operational classification of leprosy and can also be used to identify individuals infected with $M$. leprae. 
The results of this study indicate the need for greater vigilance in the health services in regions with high leprosy endemicity because many cases may currently be undiagnosed. These undiagnosed cases likely contribute to the continued transmission of $M$. leprae and maintenance of leprosy as an ongoing health concern.

\begin{abstract}
Abbreviations
POC: Point-of-care; PGL-1: Phenolic glycolipid-1; NDO-HSA: Natural disaccharide linked to human serum albumin via an octyl; IgG: Immunoglobulin G; LID-1: Leprosy IDRI Diagnostic-1; IgM: Immunoglobulin M; NDO-LID: Natural disaccharide octyl - leprosy IDRI diagnostic 1; LL: Lepromatous;

Tा: Tuberculoid; BI: Bacterial index; MB: Multibacillary; BCG: Bacillus Calmette-Guérin; BT: Borderline - tuberculoid; BB: Mid - borderline; BL: Borderline - lepromatous; PB: Paucibacillary; HHC: Household contacts; ELISA: Enzyme-linked immunosorbent assay; OPD: Ortho-phenylenediamine; El: ELISA index; OD: Optical density; H: Kruskal-Wallis one-way coefficient; rho: Spearman coefficient; GPop: General population; K: Kappa coefficient.
\end{abstract}

\section{Competing interests}

The authors declare that they have no competing interests.

\section{Authors' contributions}

ACOCF participated in the conception, design, data collection, analysis and interpretation of the data and wrote the manuscript. APMC participated in the design, data collection and data analysis and helped to draft the manuscript. SA participated in the data collection and data analysis and helped to draft the manuscript. LRG, IMBG and RCO participated in the interpretation of data and helped to draft and critically revise the manuscript. AMMM and HCT participated in the analysis and interpretation of data and helped to critically revise the manuscript. MSD participated in the critical revision of the manuscript, helped to draft the manuscript and provided the LID-1, NDO-LID and NDO-HSA antigens. FCFL conceived the study, participated in the study design and interpretation of data and critically revised the manuscript. All authors read and approved the final manuscript.

\section{Acknowledgments}

We thank the Coordenação de Aperfeiçoamento de Pessoal de Nível Superior (CAPES), Brazil for the Senior Research Fellowship to RCO and Conselho Nacional de Desenvolvimento Científico e Tecnológico (CNPq), Brazil for the PhD Scholarship Sandwich (SWE) to ACOCF. We thank the Brazilian funding agencies for the CNPq awarded to the Laboratório de Hanseníase da Fundação Oswaldo Cruz (FIOCRUZ) and for funding from the Fundação de Amparo à Pesquisa do Estado de Minas Gerais (FAPEMIG) and from the Instituto Nacional de Ciência e Tecnologia em Doenças Tropicais (INCT-DT). We thank the members of the research group "Núcleo de Estudos e Pesquisas em Hanseníase - NEPHANS" for their participation in data collection. We thank the researchers from the Centro de Tecnologia Celular e Imunologia Aplicada at the Universidade Federal de Juiz de Fora and the Centro de Referência Nacional de Dermatologia Sanitária e Hanseníase (CREDESH) at the Universidade Federal de Uberlândia for helping in the data analysis and providing the native PGL-1 antigen. We also thank American Leprosy Mission who provided funding to the Infectious Disease Research Institute (IDRI).

\section{Author details}

'Department of Basic Nursing, Faculdade de Enfermagem, Universidade Federal de Juiz de Fora - UFJF, Juiz de Fora, MG, Brazil. ${ }^{2}$ Postgraduate Program in Nursing, Escola de Enfermagem, Universidade Federal de Minas Gerais - UFMG, Belo Horizonte, MG, Brazil. ${ }^{3}$ National Reference Center for Sanitary Dermatology and Leprosy - CREDESH, Hospital das Clínicas, Universidade Federal de Uberlandia - UFU, Uberlândia, MG, Brazil. ${ }^{4}$ Institute of Genetics and Biochemistry, Universidade Federal de Uberlandia - UFU, Uberlândia, MG, Brazil. ${ }^{5}$ Department of Medical Microbiology and Immunology, University of California-Davis, Davis, CA, USA. ${ }^{6}$ Postgraduate Program in Biological Science - Immunology and Infectious Parasitic Disease, Instituto de Ciências Biológicas, Universidade Federal de Juiz de Fora - UFJF, Juiz de Fora, MG, Brazil. ${ }^{7}$ Department of Parasitology, Microbiology and Immunology, Instituto de Ciências Biológicas, Universidade Federal de Juiz de Fora UFJF, Juiz de Fora, MG, Brazil. Infectious Disease Research Institute, Seattle,
WA, USA. ${ }^{9}$ Laboratory of Cellular and Molecular Immunology, Centro de Pesquisas René Rachou - CPqRR, Fundação Oswaldo Cruz - FIOCRUZ, Belo Horizonte, MG, Brazil. ${ }^{10}$ Laboratory of Immunology, Núcleo de Pesquisas em Ciências Biológicas, Universidade Federal de Ouro Preto - UFOP, Ouro Preto, MG, Brazil. "1"Instituto Nacional de Ciência e Tecnologia em Doenças Tropicais - INCT-DT, Belo Horizonte, Brazil. ${ }^{12}$ Department of Maternal and Child Nursing and Public Health, Escola de Enfermagem, Universidade Federal de Minas Gerais - UFMG, Belo Horizonte, MG, Brazil.

Received: 3 November 2014 Accepted: 21 May 2015

Published online: 30 May 2015

\section{References}

1. Duthie MS, Raychaudhuri R, Tutterrow YL, Misquith A, Bowman J, Casey A, et al. A rapid ELISA for the diagnosis of MB leprosy based on complementary detection of antibodies against a novel protein-glycolipid conjugate. Diagn Microbiol Infect Dis. 2014;79(2):233-9.

2. Stefani MMA. Challenges in the post genomic era for the development of tests for leprosy diagnosis. Rev Soc Bras Med Trop. 2008;41(supl II):89-94.

3. Lobato J, Costa MP, Reis EM, Gonçalves MA, Spencer JS, Brennan PJ, et al. Comparison of three immunological tests for leprosy diagnosis and detection of subclinical infection. Lepr Rev. 2011;82(2):389-401.

4. Duthie MS, Goto W, Ireton GC, Reece ST, Cardoso LP, Martelli CMT, et al. Use of protein antigens for early serological diagnosis of leprosy. Clin Vaccine Immunol. 2007;14:1400-8.

5. Duthie MS, Hay MN, Rada EM, Convit J, Ito L, Oyafuso LK, et al. Specific IgG antibody responses may be used to monitor leprosy treatment efficacy and as recurrence prognostic markers. Eur J Clin Microbiol Infect Dis. 2011;30(10):1257-65

6. Cardoso LP, Dias RF, Freitas AA, Hungria EM, Oliveira RM, Collovati M, et al. Development of a quantitative rapid diagnostic test for multibacillary leprosy using smart phone technology. BMC Infect Dis. 2013;13:497.

7. Barreto JG, Bisanzio D, Guimarães LS, Spencer JS, Vazquez-Prokopec GM, Kitron U, et al. Spatial Analysis Spotlighting Early Childhood Leprosy Transmission in a Hyperendemic Municipality of the Brazilian Amazon Region. PLoS Negl Trop Dis. 2014;8:e2665.

8. Araújo S, Lobato J, Reis EM, Souza DOB, Gonçalves MA, Costa AV, et al. Unveiling healthy carriers and subclinical infections among household contacts of leprosy patients who play potential roles in the disease chain of transmission. Mem Inst Oswaldo Cruz. 2012;107 Suppl 1:55-9.

9. Goulart IMB, Souza DOB, Marques CR, Pimenta VL, Gonçalves MA, Goulart LR. Risk and Protective Factors for Leprosy Development Determined by Epidemiological Surveillance of Household Contacts. Clin Vaccine Immunol. 2008;15(1):101-5.

10. Minas Gerais. Secretaria de Estado da Saúde de (SES). State Seminar of Evaluation Leprosy. Belo Horizonte: SES/MG; 2013.

11. Ridley DS, Jopling WH. Classification of leprosy according to immunity: a five group system. Int J Lepr Other Mycobact Dis. 1966;34(3):255-73.

12. World Health Organization. Chemotherapy of leprosy for control programmes. Geneva, Switzerland: WHO Press; 1982. p. 675. 40.

13. Fabri ACOC, Carvalho APM, Cardoso VG, Abreu MNS, Lana FCF. The use of household survey in leprosy endemic areas. J Dermatol Nurses Assoc. 2014;6(3):126-31.

14. Corran PH, Cook J, Lynch C, Leendertse H, Manjurano A, Griffin J, et al. Dried blood spots as a source of anti-malarial antibodies for epidemiological studies. Malar J. 2008;7:1957.

15. Buhrer-Sekula S, Beers SV, Oskam L, Lecco R, Madeira ES, Dutra MAL, et al. The relation between seroprevalence of antibodies against phenolic glycolipid-I among school children and leprosy endemicity in Brazil. Rev Soc Bras Med Trop. 2008;41 Suppl 2:81-8.

16. Landis JR, Koch GG. The measurement of observer agreement for categorical. Biometrics. 1977;33(1):159-74.

17. Duthie MS, Truman RW, Goto W, O'Donnell J, Hay MN, Spencer JS, et al. Insight toward early diagnosis of leprosy through analysis of the developing antibody responses of Mycobacterium leprae-infected armadillos. Clin Vaccine Immunol. 2011;18(2):254-9.

18. Duthie MS1, Balagon MF, Maghanoy A, Orcullo FM, Cang M, Dias RF, et al. Rapid quantitative serological test for detection of infection with mycobacterium leprae, the causative agent of leprosy. J Clin Microbiol. 2014;52(2):613-9. 
19. Spencer JS, Brennan PJ. The role of Mycobacterium leprae phenolic glycolipid I (PGL-I) in serodiagnosis and in the pathogenesis of leprosy. Lepr Rev. 2011;82(4):344-57.

20. Souza MM, Netto EM, Nakatani M, Duthie MS. Utility of recombinant proteins LID-1 and PADL in screening for Mycobacterium leprae infection and leprosy. Trans R Soc Trop Med Hyg. 2014;108(8):495-501.

21. Brasil MTLRF, Oliveira LR, Rímoli NS, Cavallari FS, Gonçalves OS, Lessa ZL, et al. Anti PGL-1 serology and the risk of leprosy in a highly endemic area in the State of São Paulo, Brazil: four-year follow-up. Rev Bras Epidemiol. 2003;6(3):262-71.

22. Moet F, Schuring R, Pahan D, Oskam L, Richardus J. The prevalence of previously undiagnosed leprosy in the general population of northwest bangladesh. PLoS Negl Trop Dis. 2008;2(2):e198.

23. Cho S, Cellona RV, Villahermosa LG, Fajardo Jr TT, Balagon MVF, Abalos RM, et al. Detection of phenolic glycolip I of Mycobacterium leprae in sera from leprosy patients before and after start of multidrug therapy. Clin Diagn Lab Immunol. 2001;8(1):138-42.

24. Schuring RP, Moet FJ, Pahan D, Richardus JH, Oskam L. Association between anti-PGL-I IgM and clinical and demographic parameters in leprosy. Lepr Rev. 2006;77(4):343-55.

\section{Submit your next manuscript to BioMed Central and take full advantage of:}

- Convenient online submission

- Thorough peer review

- No space constraints or color figure charges

- Immediate publication on acceptance

- Inclusion in PubMed, CAS, Scopus and Google Scholar

- Research which is freely available for redistribution 